\title{
Double VTs associated with an anatomical isthmus identified by a CT-derived channel
}

Masateru Takigawa ${ }^{1}$, Ruairidh Martin ${ }^{2}$, Takeshi Kitamura ${ }^{3}$, Hubert Cochet $^{4}$, Frederic Sacher $^{5}$, and Pierre Jais ${ }^{1}$

${ }^{1}$ LIRYC institute, Université de Bordeaux, Centre Hospitalier universitaire de Bordeaux, France

${ }^{2}$ Hopital Haut-Leveque

${ }^{3}$ IHU LIRYC, University of Bordeaux, CHU de Bordeaux

${ }^{4} \mathrm{CHU} /$ Université de Bordeaux

${ }^{5}$ Bordeaux University Hospital

June 22, 2020

\begin{abstract}
We demonstrated a 47 year-old woman with ischemic-VT with repetitive ICD-shocks, requiring ablation. Pre-procedural CT demonstrated a single anatomical channel on the inferior-basal infarcted area between $<3 \mathrm{~mm}$ wall thinning area and the mitral annulus, which suggested the circuit of two VTs observed. Additionally, distribution of $<2 \mathrm{~mm}$ and $<3 \mathrm{~mm}$ scars can explain the mechanism of the variation of QRS morphology and S-QRS interval during entrainment. Ablation on this region resulted in no VT- inducibility and absence of any VTs for 2yrs. CT wall thinning data may allow us to understand the mechanism and circuit of VT and aid VT ablation procedures.
\end{abstract}

Figure 1

(A)

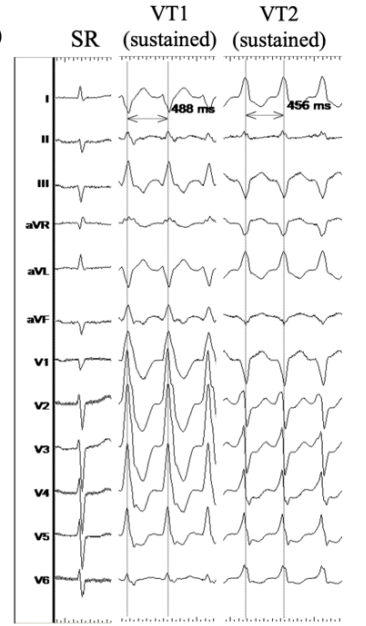

(B)

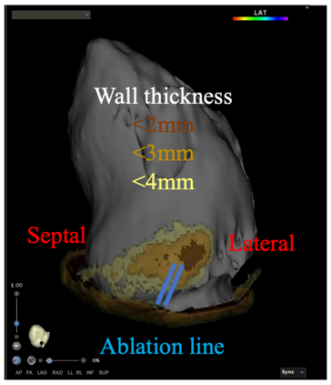

(C)

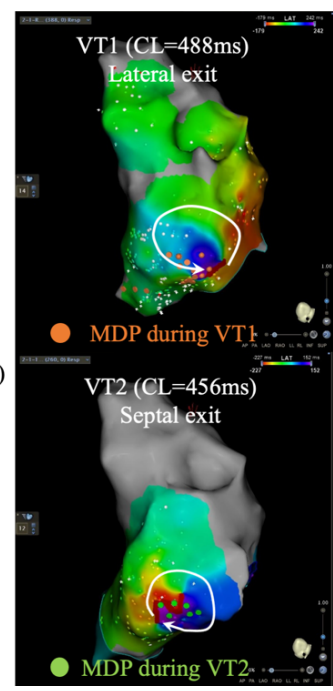



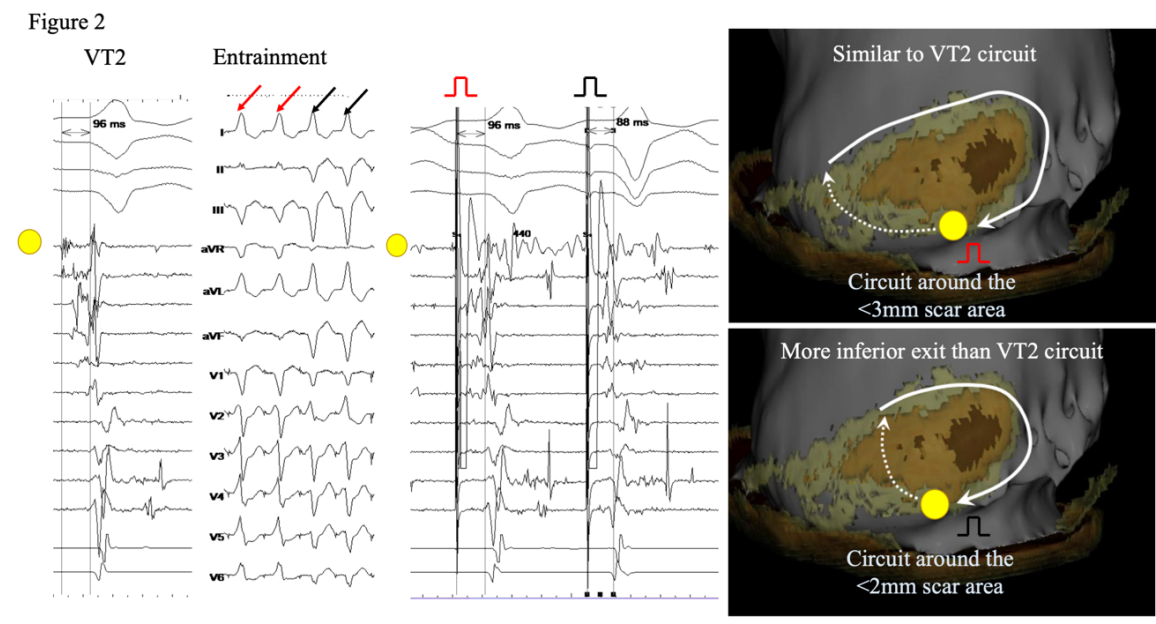

\section{Figure legends}

\section{Figure 1: ECG and Circuit of clinical VTs}

12-lead ECG of VT1 and VT2 (A). Distribution of wall thinning areas based on CT (B). VT1 rotates the scar area in clockwise direction (C) and VT2 rotates clockwisely.

\section{Figure 2: Entrainment pacing during VT2}

\section{References}

1. Stevenson WG, Sager PT, Friedman PL. Entrainment techniques for mapping atrial and ventricular tachycardias. J Cardiovasc Electrophysiol. 1995;6:201-216.

2. de Chillou C, Lacroix D, Klug D, Magnin-Poull I, Marquié C, Messier M et al. Isthmus characteristics of reentrant ventricular tachycardia after myocardial infarction. Circulation. 2002;105:726-731

3. Bogun F, Li YG, Groenefeld G, Hohnloser SH, Schuger C, Oral H, et al. Prevalence of a shared isthmus in postinfarction patients with pleiomorphic, hemodynamically tolerated ventricular tachycardias.

J Cardiovasc Electrophysiol. 2002 Mar;13:237-41. 